\title{
Optimal Charging of Electric Vehicles Taking Distribution Network Constraints Into Account
}

\author{
Julian de Hoog, Member, IEEE, Tansu Alpcan, Senior Member, IEEE, Marcus Brazil, \\ Doreen Anne Thomas, Senior Member, IEEE, and Iven Mareels, Fellow, IEEE
}

\begin{abstract}
The increasing uptake of electric vehicles suggests that vehicle charging will have a significant impact on the electricity grid. Finding ways to shift this charging to off-peak periods has been recognized as a key challenge for integration of electric vehicles into the electricity grid on a large scale. In this paper, electric vehicle charging is formulated as a receding horizon optimization problem that takes into account the present and anticipated constraints of the distribution network over a finite charging horizon. The constraint set includes transformer and line limitations, phase unbalance, and voltage stability within the network. By using a linear approximation of voltage drop within the network, the problem solution may be computed repeatedly in near real time, and thereby take into account the dynamic nature of changing demand and vehicle arrival and departure. It is shown that this linear approximation of the network constraints is quick to compute, while still ensuring that network constraints are respected. The approach is demonstrated on a validated model of a real network via simulations that use real vehicle travel profiles and real demand data. Using the optimal charging method, high percentages of vehicle uptake can be sustained in existing networks without requiring any further network upgrades, leading to more efficient use of existing assets and savings for the consumer.
\end{abstract}

Index Terms-Distribution networks, electric vehicles, grid impacts, optimization, receding horizon, smart charging.

\section{NOMENCLATURE}

$\mathcal{H} \quad$ Set of houses in the network $\{1 \ldots H\}$.

$\mathcal{K} \quad$ Set of electric vehicles, each associated with a house, $\mathcal{K} \subset \mathcal{H}$.

$\mathcal{T} \quad$ Set of discrete time intervals in charging horizon $\{1 \ldots T\}$.

$x_{k, t} \quad$ Current (A) drawn by vehicle $k$ at time $t$.

$x_{h, t} \quad$ Current (A) drawn by household $h$ at time $t$.

$x_{h, t}^{\text {tot }} \quad$ Total current (A) drawn at household $h$ (from both household and vehicle).

Manuscript received November 04, 2013; revised March 06, 2014; accepted April 05, 2014. This work was supported by a Linkage Grant supported by the Australian Research Council, Better Place Australia, and Senergy Australia. Paper no. TPWRS-01408-2013.

J. de Hoog and D. A. Thomas are with the Department of Mechanical Engineering, The University of Melbourne, Australia (e-mail: julian. dehoog@unimelb.edu.au).

T. Alpcan, M. Brazil, and I. Mareels are with the Department of Electrical and Electronic Engineering, The University of Melbourne, Australia.

Color versions of one or more of the figures in this paper are available online at http://ieeexplore.ieee.org.

Digital Object Identifier 10.1109/TPWRS.2014.2318293
$x_{\phi, t}$
Total current (A) drawn by all single-phase loads on phase $\phi \in\{A, B, C\}$.
$s_{k}(t) \quad$ Stored energy $(\mathrm{kWh})$ of vehicle $k$ at time $t$.
$\tau \quad$ Charging efficiency factor.
$V_{T x} \quad$ Source voltage (V) at distribution transformer, phase to neutral.
$s^{\max } \quad$ Maximum allowed stored energy (kWh).
$P_{T x}^{\max } \quad$ Nominal power rating (kVA) of distribution transformer.
$x_{\phi}^{\max } \quad$ Current rating (A) of feeder backbone cable, phase $\phi \in\{A, B, C\}$.
$x_{k}^{\max } \quad$ Current rating (A) of service line connecting house $k$ to feeder.
$V_{h, t}^{\text {drop }} \quad$ Difference between voltage at transformer and voltage at house $h$ at time $t(\mathrm{~V})$, phase to neutral.

\section{INTRODUCTION}

$\mathbf{E}$ LECTRIC vehicles (EVs) are being pushed by governments around the world as an alternative to fossil fuel based transport. As a result, EV market share is starting to increase: a recent report by the U.S. Department of Energy shows that plug-in vehicle sales are more than double those of hybrids when comparing the same stages of the technology life cycle [1].

The charging of so many electric vehicles puts an additional strain on the existing electricity grid. Since people are likely to plug in when they arrive at home, there is a risk that vehicle charging will coincide with peak demand. If vehicle charging is not controlled, adverse impacts on the distribution network are expected: power demand may exceed distribution transformer ratings; line current may exceed line ratings; phase unbalance may lead to excessive current in the neutral line; and voltages at customers' points of connection may fall outside required levels [2]-[5]. However, these impacts can be alleviated if vehicle charging is shifted to a time when there is more capacity in the network, such as overnight. An effective shifting of EV charging load to off-peak periods means that existing networks can be used considerably more efficiently, reducing the need for network upgrades and ultimately benefitting the consumer.

The "smart charging" problem is well studied, and many approaches have been proposed to achieve this behaviour. Several studies pursue distributed methods, in which charge points or vehicles make individual decisions on whether to charge or not using local information [5]-[7]. The advantage of such methods is that they usually do not require any particular metering or 
communication infrastructure. The disadvantages are that they may not be able to fully make use of available system capacity due to limited knowledge of the network's current state, and that they may be difficult to regulate.

Other studies use full network state information and control vehicle charge rates centrally [5], [8]-[14]. One such approach uses both quadratic and dynamic programming to minimize network losses and voltage deviations [8]. Across a variety of case studies, it is shown that this approach reduces losses, improves voltage stability, and decreases peak load when compared with uncontrolled charging. The relationships between losses, load factor, and load variance are further explored in [9]. Reference [10] proposes a way of expressing centralized EV charging using results from recent optimal power flow studies. An optimal problem formulation aims to minimize generation and charging costs while satisfying all the constraints posed by the network, and the optimal power flow problem takes into account both elastic and inelastic loads. Reference [12] similarly tries to maximize the revenue that a distribution network operator might have in response to network operating costs and fluctuating wholesale prices. Network voltages are taken into account by iteratively solving a linear program until all voltages fall within required levels. Reference [13] uses a two-stage optimization approach, in which the minimum required peak is determined in the first stage, and load fluctuation is minimized in the second stage by choosing controllable vehicle charge rates within the allowed peak load. The focus is on cost-benefit analysis and distribution network constraints are not considered. A model predictive control framework is proposed in [14] that minimizes the cost of energy consumption. Network demand is limited via tracking of a reference load profile defined by the grid operator.

A further centralized approach uses linear programming to maximize the total vehicle charging power that the distribution network allows, while formulating network limitations as constraints [11]. Sensitivity analysis is applied to determine how sensitive the voltage of each node in the network is to the addition of EV load. The optimal solution allows for the modelled network to sustain an EV penetration of 50\%, as compared with only $16 \%$ in the uncontrolled case.

However, many existing methods suffer from one or more of the following drawbacks: 1) The charging period is often modelled as a static time interval (typically overnight), in which vehicles do not arrive or depart; 2) distribution network constraints, in particular voltage drop, are often estimated, or only included in an indirect way; and 3) phase unbalance is often ignored, and limitations are expressed for the network as a whole when instead they should be expressed on a phase-by-phase basis.

In reality, vehicles may arrive and depart unexpectedly. Distribution network constraints can already be breached at very low vehicle uptake rates: in previous work we have found that with uncontrolled charging there is a risk of voltage dropping below distribution code limitations at EV penetrations of only $10 \%$ [5] (a finding that is in line with several studies elsewhere, e.g., [3], [11]). In the networks we have studied (where each house is connected single-phase), phase unbalance has been a major factor, with the most heavily loaded phase sometimes having close to double the total load of the least loaded phase.
In this paper we build on existing work by addressing these concerns, and provide a novel optimal smart charging algorithm that allows large numbers of vehicles to be charged without adverse effects on the network. We express smart charging as an efficient linear optimization problem that takes into account both the present and anticipated constraints of the distribution network over the full finite charging horizon. Recalculating our solution in discrete intervals allows for the dynamic nature of vehicle arrival/departure to be accommodated. We model voltage drop explicitly as a linear constraint, on a phase-by-phase basis, in every lateral of the network. We maintain separate loading constraints for each phase.

Using this framework, we examine two possible objectives: in the first, we aim to provide as much charging power to the vehicles as the network will allow. In the second, we take fluctuations in the electricity price into account and aim to charge all vehicles within a specified time period at minimum cost.

Our methods are implemented and simulated on a validated model of a real distribution network, using real travel profiles to simulate EV behavior and real demand profiles obtained in the network we are modelling. While we use linear approximations when solving our optimization problem, the simulations are run in a fully complex, unbalanced, three-phase load flow scenario.

It is shown that linear approximation in this manner is an effective, fast way to find a charge scheduling solution. It is also shown that existing networks can sustain high penetration rates of electric vehicles, without significant additional investment into network assets required.

\section{UNDERLYING MODEL}

\section{A. Preliminaries}

We aim to determine the charging rates of vehicles in a radial distribution network served by one transformer. We assume that vehicles' charging rates may be controlled centrally, and may be set to any value within a given continuous range. We consider this a realistic assumption since this was recently demonstrated in the Australian Victoria Electric Vehicle Trial as part of a pilot load control project [15]. We further assume that the network operator has access to the following information:

- network size (number of houses);

- network structure (including line segment lengths and individual phase connections);

- line specifications (impedance per $\mathrm{km}$, nominal current ratings);

- transformer specifications (nominal power rating).

This information may be used by a central controller to determine the best set of charging rates for all vehicles currently connected, so that they may charge in a way that maximizes a given objective while not violating any constraints in the network. In making this decision, we are looking not just at the current point in time, but at the best possible solution for a finite future charging horizon in discrete intervals. Since underlying conditions may change unexpectedly (such as vehicles arriving or departing), the solution for the full charging horizon is recomputed after each discrete time interval.

The distribution networks we have studied have had a high power factor. Fig. 1 shows data obtained in the network presented in this paper; more than $99 \%$ of data points have power 


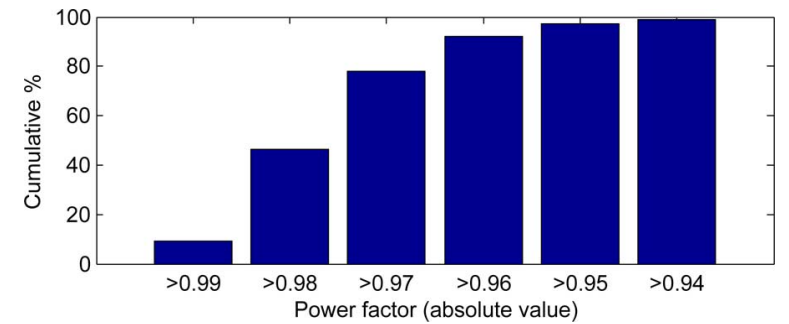

Fig. 1. Cumulative frequency histogram of power factor measurements in the data set provided by our utility partner. This histogram reflects 43317 data points (14 439 for each phase) gathered in the period 17-27 August 2012 in the network described in Section VII-A.

factor 0.95 or higher. We therefore consider it a reasonable simplification to use a DC-equivalent model of our distribution network when formulating our optimization problem. This is common practice when the angle between source voltage and load voltage is very small [16]. By doing this we keep our constraint set linear, and in most cases it leads to conservative constraints. However, when we run our simulations to examine our solutions (Section VII), we conduct unbalanced, three-phase AC load flow analyses in fully realistic scenarios that have been validated to have a high correlation to reality.

\section{B. Notation}

Our notation is as outlined before Section I. Let $\mathcal{H}$ be the set of $H$ houses in the network. (In Australia, houses are typically connected single-phase.) Let $\mathcal{K} \subset \mathcal{H}$ be a set of $K$ households owning electric vehicles. We assume charging decisions can be made in discrete time intervals; let $\mathcal{T}$ be the discretized charging horizon having $T$ intervals.

We denote current at point of connection $h$ at time $t$ as $x_{h, t}$ (current due to household load) and $x_{k, t}$ (current due to vehicle load). Total current at household $h$ at time $t$ is

$$
x_{h, t}^{\mathrm{tot}}=x_{h, t}+x_{k, t} .
$$

We model the network as a three-phase wye-connected system. Total current on a given phase is the sum of all currents at any points of connection on that phase:

$$
x_{\phi, t}=\sum_{h \in \phi} x_{h, t}^{\mathrm{tot}}, \quad \phi \in\{A, B, C\} .
$$

\section{Vehicle Batteries}

We use stored energy ( $\mathrm{kWh}$ ) as a measure of how charged the vehicle batteries are. The stored energy of the battery of the $k$ th vehicle at time $t$ must satisfy $0 \leq s_{k}(t) \leq s^{\max }$. We estimate the future stored energy of a battery using the following:

$$
s_{k}(t)=s_{k}(t-1)+\tau V_{\text {nom }} x_{k, t} \Delta t, \quad k \in \mathcal{K}, t \in \mathcal{T}
$$

where $V_{\text {nom }}$ is the nominal voltage of the grid, $x_{k, t}$ is current supplied to the vehicle, $\Delta t$ is the size of our discretized time interval and $\tau$ is an efficiency factor (we use 0.9) that takes into account energy lost due to AC/DC conversion and cooling. The voltage at point of connection will usually not vary beyond 5\% (limits are imposed by the Electricity Distribution Code [17]), so we consider this a sufficiently accurate way of estimating a battery's stored energy over future time intervals. If necessary, a conservative nominal voltage may be chosen so that the stored energy of a battery at a future time interval is not overestimated.

We assume that batteries can be charged at variable rates, provided minimum and maximum rates are not exceeded (as demonstrated in a pilot load control trial using commercially available electric vehicles [15]).

Vehicles arrive and depart independently of one another. Each vehicle has a charging horizon, with a target of being fully charged at time $T_{k}$.

\section{DECISION VARIABLES}

In principle, the purpose of our optimization problem is to determine the amount of power delivered to the grid-connected electric vehicles. However, because EV charge points follow the voltage from the grid, which is highly regulated, a charge current decision is essentially equivalent (in fact, the $\mathrm{J} 1772$ standard for car battery charging is specified in terms of charging current, not power [18]). In view of this, we choose the currents supplied to each grid-connected vehicle as our decision variables. This further allows us to keep our problem formulation linear in our decision variables.

Our decision variables are therefore the currents supplied to all charging vehicles over all intervals in the charging horizon, which may be denoted by the matrix

$$
x=\left(x_{k, t}\right)=\left(\begin{array}{ccc}
x_{1,1} & \ldots & x_{1, T} \\
\vdots & & \vdots \\
x_{K, 1} & \ldots & x_{K, T}
\end{array}\right)
$$

where $k \in \mathcal{K}$ and $t \in \mathcal{T}$. We can rewrite matrix $x$ as a vector by using its column vectors.

The full solution to our problem is recomputed at each interval (e.g., every $5 \mathrm{~min}$ ). The number of decision variables may change from one interval to the next, depending on whether vehicles have arrived or departed.

\section{System CONSTRAINTS}

Our full set of system constraints at any time interval may be written in the standard format $A x \leq b$, where the matrix $A$ and the vector $b$ result from the grid and battery conditions at each interval in our horizon. At any point in time there may be many vehicles in the network, and for each of these vehicles charge rates must be chosen for all intervals within a given future horizon. As a result, the number of decision variables and constraints can grow quickly (both often in the thousands for our case study). We therefore make a series of approximations that allow us to express the constraints in the distribution network in a linear form. This ensures the optimization problem can be solved very quickly, and makes our method suitable for near-real-time decisions. Our simulation results (Section VII), which are conducted in a fully complex, unbalanced, 3-phase, validated system, suggest that our approximations are justified since this way of formulating the problem successfully avoids any network constraints being violated. The effects of linearization are also further discussed in Section VII-E.

\section{A. Nominal Power Rating of Transformer}

Transformers have a nominal power rating $P_{T x}^{\max }$ that should not be significantly exceeded - if it is, transformer lifetime is reduced. However, transformers often run at $130 \%$ of $P_{T x}^{\max }$ or 
more, which is sustainable as long as there is an accompanying cooling period. We express this constraint on a phase-by-phase basis:

$$
V_{T x} x_{\phi, t} \leq \frac{1}{3} P_{T x}^{\max } \times 130 \%, \quad \phi \in\{A, B, C\}
$$

where $V_{T x}$ is the phase-to-neutral voltage at transformer. Since we are capping total power for each phase instead of for the system as a whole, these are conservative constraints.

\section{B. Nominal Current Ratings of Lines}

Power lines have a variety of limitations, the most important of which is current rating. If this is exceeded, cables can be damaged. Let cable $i$ have current rating $x_{i}^{\max }$. In the networks we have studied, backbone and service lines typically have different specifications. Thus we introduce separate constraints for each phase of the backbone, and further add individual constraints for every service line in the network:

$$
\begin{array}{rll}
\text { Backbone : } & x_{\phi, t} \leq x_{\phi}^{\max }, & \phi \in\{A, B, C\} \\
\text { Service Lines : } & x_{k, t}^{\text {tot }} \leq x_{k}^{\max }, & \forall k \in \mathcal{K} .
\end{array}
$$

\section{Voltage Drop}

Voltage must be maintained within lower and upper limits at every point of connection. ${ }^{1}$ If these limits are not respected, household loads can be adversely affected.

Consider the simplified network in Fig. 2, where each load $S_{i}$ is a combination of household and electric vehicle loads, and all are on the same phase. The total voltage drop $V^{\text {drop }}$ from transformer to house 4 can be approximated by considering a DC-equivalent circuit:

$$
V_{4}^{\text {drop }}=\left(I_{2}+I_{3}+I_{4}\right) z_{12}+\left(I_{3}+I_{4}\right) z_{23}+I_{4} z_{34}
$$

and in general at house $h$

$$
V_{h}^{\mathrm{drop}}=\sum_{j=1}^{h}\left(I_{j} \sum_{k=1}^{j} z_{k-1, k}\right) .
$$

Since $I_{j}$ are a combination of the existing household and electric vehicle load, and since we are modelling the network as mainly resistive (using only real power), this becomes a linear expression. We can now formulate a constraint at every house $h$ at every future time interval $t$ to ensure that voltage is high enough:

$$
V_{T x}-V_{h, t}^{\text {drop }}>V^{\text {min }} .
$$

Since networks may have multiple laterals, we must consider what happens when there is a split. Consider the simplified network in Fig. 3. The voltages in each lateral, at $S_{3}$ and $S_{4}$, are not independent since they share an impedance $z_{12}$. To ensure that

\footnotetext{
${ }^{1}$ In Australia, voltages at point of connection must be maintained at $230 \mathrm{~V}$, $+10 \% /-6 \%$, i.e., in the range $216 \mathrm{~V}-253 \mathrm{~V}$ [17].
}

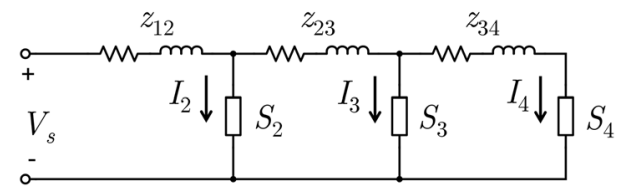

Fig. 2. Simple network.

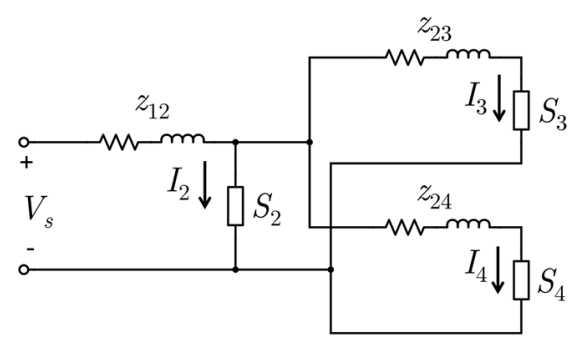

Fig. 3. Simple network with two branches.

voltage remains within limits at all locations in the network, we consider the worst case when expressing this as a constraint:

$$
V_{T x}-\max \left(V_{12}+V_{23}, V_{12}+V_{24}\right)>V^{\min }
$$

where $V_{i j}$ represents the voltage drop across $z_{i j}$. This can be generalized to

$$
V_{T x}-\sum\left[V_{a, b}^{\mathrm{drop}}\right]_{h, t}>V^{\mathrm{min}}
$$

where $\left[V_{a, b}^{\mathrm{drop}}\right]_{h, t}$ are all piecewise voltage drops from source to house $h$ at time $t$. In other words, at each interval, each house will have its own unique constraint that is still linear in the currents through all other houses and vehicles in the network. The performance of this linearization is explored as part of our simulation case study in Section VII-E.

\section{Phase Unbalance}

Phase unbalance can lead to overheating of motors, may have negative effects on electrical equipment, and leads to higher current in the neutral (which in turns contributes to voltage drop).

Phase unbalance is typically expressed in terms of percent negative sequence voltage. However, to keep our constraint set linear, we express phase unbalance in terms of $p$, the percent deviation from average phase load:

$$
\frac{\left|x_{\phi, t}-\frac{1}{3}\left(x_{A, t}+x_{B, t}+x_{C, t}\right)\right|}{\frac{1}{3}\left(x_{A, t}+x_{B, t}+x_{C, t}\right)}<p, \quad \phi \in\{A, B, C\} .
$$

Note that these constraints are linear when we multiply both sides of the equation by the denominator.

\section{E. Battery}

It is important not to exceed a battery's maximum capacity. This can be expressed by the following constraint:

$$
s_{k}(t)<s^{\max } \quad \forall t \in[0, T] .
$$


Batteries further have minimum and maximum possible charging currents that should be respected to protect the battery and ensure efficient charging:

$$
x^{\min }<x_{k, t}<x^{\max } .
$$

\section{F. Charging Targets}

Each vehicle has a target of reaching at least $95 \%$ of its maximum stored energy within a finite, specific time $T_{k}$ from the moment it starts charging:

$$
s_{k}(0)+\sum_{t=0}^{T_{k}} \tau x_{k, t} V_{\mathrm{nom}} \Delta t>0.95 \times s^{\max } .
$$

\section{OBJeCtive FunCtions}

We now examine two possible objective functions:

\section{A. Greedy Charging (GC)}

Our first objective is greedy: we want to only maximize the stored energy of all the vehicles without considering pricing or fairness issues:

$$
\max _{x_{k, t}} \sum_{k=1}^{K} s_{k}(T) \quad \text { s.t. } A x \leq b, \quad \forall k, t .
$$

Using the battery state evolution (1), the problem may be equivalently expressed as

$$
\max _{x_{k, t}} \sum_{k=1}^{K} \sum_{t=0}^{T-1} x_{k, t} \quad \text { s.t. } A x \leq b, \quad \forall k, t .
$$

In other words, we want to provide as much possible charging current as the network will allow.

\section{B. Greedy Charging With Pricing (GCP)}

Our second objective is to minimize the cost of charging (a problem of interest, for example, to a charging provider or aggregator). We use the dynamically changing spot price of electricity, $p(t)$, as a parameter:

$$
\min _{x_{k, t}} \sum_{k=1}^{K} \sum_{t=0}^{T-1} p(t) x_{k, t} \quad \text { s.t. } A x \leq b, \quad \forall k, t .
$$

Since we have charging targets as a constraint (2), our solution must contain non-zero values for $x_{k, t}$. However, this objective means that higher charge rates are chosen when the spot price is low.

\section{PRoBlem COMPLEXITY}

In this section we explore the complexity of formulating the problem using decision variables as described in Section III and constraints as described in Section IV. We assume as before that there are $H$ houses having $K$ charging vehicles, making decisions over a horizon having $T$ intervals.

Using our problem formulation, there will be $K \times T$ decision variables. The number of constraints is summarized in Table I; the only constraint that may not be immediately clear is phase unbalance. For this, we need to ensure that each phase does not deviate from average phase load, and since there are absolute
TABLE I

Number of Constraints for $H$ Houses Having $K$ Charging Vehicles OVER A CHARGING HORIZON OF $T$ INTERVALS

\begin{tabular}{|c|l|c|}
\hline Section & \multicolumn{1}{|c|}{ Constraint Type } & Num. constraints \\
\hline V-A & Transformer & $3 \times T$ \\
V-B & Lines - Backbone & $3 \times T$ \\
V-B & Lines - Service & $K \times T$ \\
V-C & Voltage Drop & $H \times T$ \\
V-D & Phase Unbalance & $6 \times T$ \\
V-E & Battery - Max Capacity & $K \times T$ \\
V-E & Battery - Max and Min Currents & $2 K \times T$ \\
V-F & Charging Targets & $K$ \\
\hline
\end{tabular}

values involved we have two constraints for each phase for each time interval. Summing the rows presented in Table I leads to a total of $(12+4 K+H) T+K$ constraints.

As an example, for the case study explored in Section VII we will have 114 houses with 57 vehicles, making decisions in 15-min intervals over an 8-h horizon (for a total of 32 intervals). In the worst case (when all vehicles are charging), there will thus be 1824 decision variables and 11385 constraints. By linearizing the constraints, this problem can be solved in a matter of seconds on a standard desktop PC using the MATLAB Optimization Toolbox. This speed of computation becomes all the more important if the method is to be applied to multiple networks, or at a higher level such as the substation.

\section{IMPLEMENTATION AND RESULTS}

In the preceding sections, several simplifications were made to allow us to keep our problem formulation and constraints linear. In this section we implement and test our solution on a validated model of a real network, and run fully complex, unbalanced, three-phase load flow analyses in each interval. Testing our solution under fully realistic conditions allows us to confirm whether our simplifications were justified or not.

\section{A. Simulator, Data, and Case Study}

Our simulations were conducted in POSSIM, ${ }^{2}$ a tool developed at The University of Melbourne for analysis of distribution networks. POSSIM is a $\mathrm{C}++$ based simulator that uses a MATLAB SimPowerSystems backend for model building and load flow analyses.

To conduct a realistic case study, we developed a model of a real suburban distribution network in Melbourne containing 114 customers [Fig. 4(a)]. Detailed demand data was provided by the network operator for each phase [Fig. 4(b)]. Individual phase connections were not provided, but could be estimated using aggregated load data. As can be seen, this is a fairly unbalanced network with 50 houses connected to phase A, 43 houses to phase $\mathrm{B}$, and 21 houses to phase $\mathrm{C}$. Each house was assigned an average load profile on a per-phase basis using the data obtained in the network, having both active and reactive power demand.

The network is served by a $300 \mathrm{kVA}$ transformer. Line impedances for both backbone and service lines were provided by the network operator, as were distances between poles and lengths of individual service lines. A validation cycle in which we compared our simulated voltages and currents to those measured in the real network concluded that on average, voltages

\footnotetext{
${ }^{2}$ POSSIM: POwer Systems SIMulator. Available at http://www.possim.org.
} 


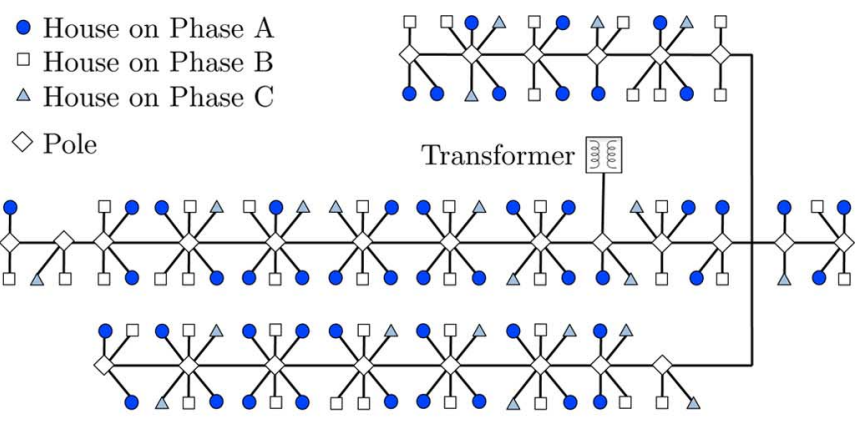

(a)

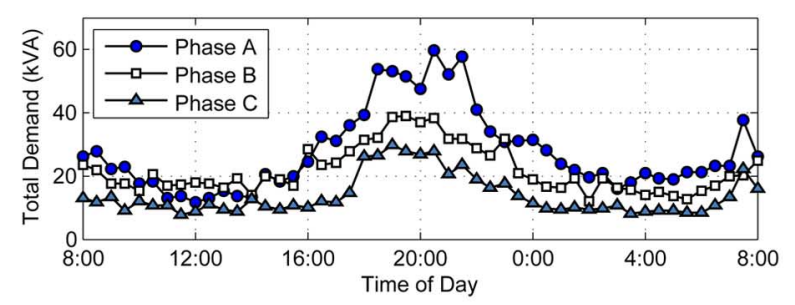

(b)

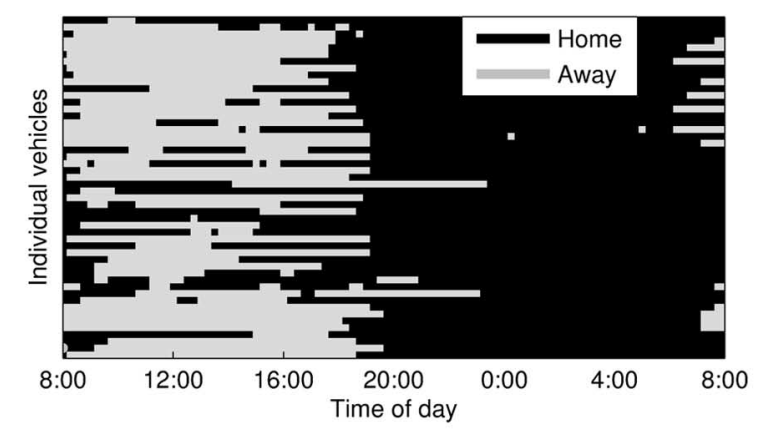

(c)

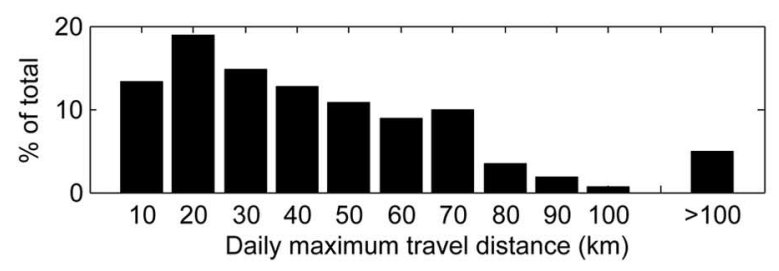

(d)

Fig. 4. Network model and data used for case study. (a) Diagram of network. (b) Household demand for a weekday in August 2012. (c) Vehicle locations (charging availability). (d) Vehicle travel distances (charging needs).

were within $1-2 \mathrm{~V}(<1 \%$ difference $)$, and currents were within 4-6 A (5-7\% difference) - in other words, a fairly close correlation between simulation and reality [19].

To model EV arrival/departure and travel distances (i.e., charging needs), we used travel survey data gathered by the Victoria Department of Transport in 2009 [20], which contains individual vehicle travel records for 24-h periods. We refined the data set of all vehicle trips to only those trips made in the local government area of our network, on weekdays, having a total travel distance of less than $160 \mathrm{~km}$ (a range in line with many commercial electric vehicles available today). This left us with 324 individual records that could be assigned, at random, individually to each vehicle in our case study. Fig. 4(c) shows when vehicles are typically at home and available for

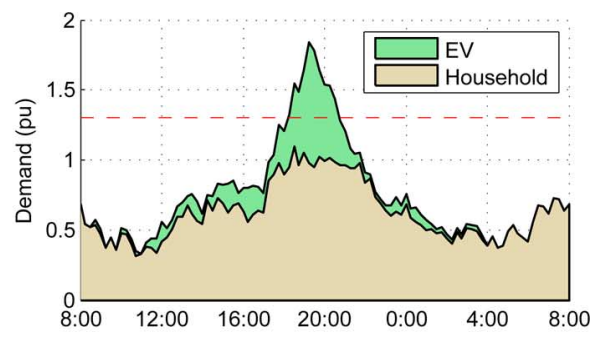

(a)

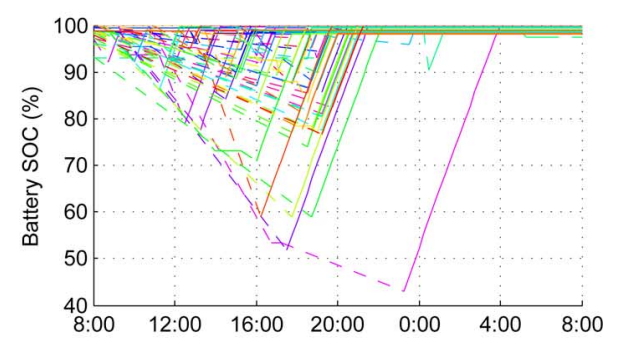

(b)

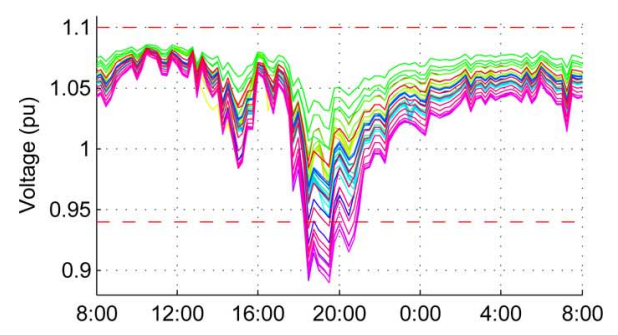

(c)

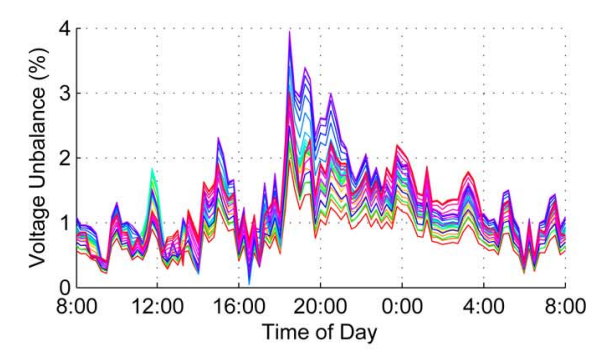

(d)

Fig. 5. Uncontrolled charging. (a) Total demand. (b) Vehicle charging rates. (c) Household voltages. (d) Unbalance.

charging, and Fig. 4(d) shows the travel distances for people in this neighborhood (average daily travel is $37.7 \mathrm{~km}$ ).

We assumed the vehicles to have a $24 \mathrm{kWh} \mathrm{Li}$-ion battery (again, similar to many of today's commercial EVs). Battery charging was modelled using a simplified Li-ion battery model that takes into account internal resistance, dynamic state-ofcharge dependent open circuit voltage, and maximum voltage. The charging process follows a constant power, constant voltage process, with a voltage limit of $4.2 \mathrm{~V}$. Battery discharge is difficult to model and depends on factors that are difficult to simulate, such as driving style. To maintain consistency across all of our runs, we assumed an average discharge rate directly proportional to distance driven. While this may not provide realistic variability in discharge from one battery to the next, it does 
TABLE II

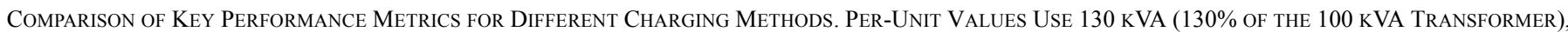
230 V (Nominal Voltage), and 350 A (Our Cables’ Maximum Current Rating)

\begin{tabular}{|l||c|c|c|}
\hline & Uncontrolled & Greedy & Greedy with Price \\
\hline Increase in peak/base ratio due to EVs (\%) & 61.3 & 18.9 & 9.3 \\
Maximum demand (pu) & 1.42 & 1.00 & 0.92 \\
Maximum cable load (pu) & 0.99 & 0.72 & 0.72 \\
Number of houses under voltage & 14 & 0 & 0 \\
Minimum voltage (pu) & 0.89 & 0.95 & 0.95 \\
Peak unbalance (\%) & 3.95 & 3.09 & 3.66 \\
Average charging rate (kW) & 3.45 & 2.36 & 0.71 \\
EVs charged to 95\% or more by 6am (\%) & 100 & 100 & 87.7 \\
Generation cost per kWh (\$) & 0.0480 & 0.0469 & 0.0433 \\
\hline
\end{tabular}

provide a good estimate of the total charging needs across the network as a whole.

As a case study, we chose a scenario in which $50 \%$ of households own an electric vehicle. While such high levels of EV ownership are unlikely to be reached for many years, we considered it a worthwhile exercise to examine the potential impacts on the network. We further used a transformer capacity of 100 kVA in our simulations - this is much tighter than the real limit of $300 \mathrm{kVA}$, but is useful for emphasizing the different behaviors of our algorithms.

\section{B. Uncontrolled Charging}

In a first set of simulations, we allowed all vehicles to charge at their maximum possible rates $(3.45 \mathrm{~kW}$ at a standard $230 \mathrm{~V}$ 15 A outlet) whenever they arrived at home, and allowed them to charge to completion. In this scenario, most vehicle charging occurs at peak demand time [Fig. 5(a)], leading to a significantly higher peak.

Fig. 5(b) presents state of charge of individual vehicles, with each line representing one vehicle. Dashed lines indicate that a vehicle is not at home. As can be seen, all vehicles charge at their maximum rates and reach full charge as quickly as possible.

Individual voltages at point of connection of all houses on phase A (the most heavily loaded phase) are shown in Fig. 5(c). Notably, during peak times voltages at many houses drop below the minimum threshold of $0.94 \mathrm{pu}$ due to additional vehicle demand.

Phase unbalance $\left(\left|V^{-}\right| /\left|V^{+}\right|\right)$is shown in Fig. 5(d), where each line represents unbalance at one pole in the network. This network is unbalanced to begin with but at peak an even higher unbalance of $4 \%$ is reached.

Key performance metrics are displayed in Table II. As can be seen, uncontrolled charging leads to a significant increase in peak/base ratio, overloading of the transformer, and voltage dropping below distribution code limits at 14 houses.

In the real network, even the high peak demand resulting from uncontrolled charging would be sustainable with a 300 kVA transformer. However, due to voltage dropping below code limits, this network would not be able to sustain a $50 \%$ penetration of electric vehicles under uncontrolled charging conditions.

\section{Greedy Charging}

In our next set of simulations, we applied the greedy charging algorithm detailed in Section V-A. The results are presented in Fig. 6 and key performance metrics are listed in Table II. There is an increase in peak demand [Fig. 6(a)], but it remains below
$130 \%$ of transformer capacity ( $100 \mathrm{kVA})$. Most vehicles can still charge at their maximum rates, but some have noticeably reduced rates at particular times in order to prevent the system exceeding any of the network constraints [Fig. 6(b)]. However, all vehicles are fully charged by their target charge goal, and in fact all vehicles are fully charged by 4:30 am. Voltages are considerably more stable than in the uncontrolled case, with no house falling below the 0.94 pu minimum threshold [Fig. 6(c)]. Phase unbalance is also noticeably improved as compared with the uncontrolled case, with a peak unbalance of 3.09\% [Fig. 6(d)]. Using the greedy charging algorithm, therefore, this network would be able to sustain a $50 \%$ electric vehicle uptake.

\section{Greedy Charging With Price Consideration}

The results of implementing our greedy charging algorithm that takes price into account (Section V-B) are shown in Fig. 7 and key performance metrics are listed in Table II. To model the electricity spot price, we used historical wholesale price data for the same day on which our demand data was collected (Fig. 8). Our look-ahead window in this case was $8 \mathrm{~h}$; it can clearly be seen that the greedy charging with price algorithm successfully identifies times of low spot price and allocates charging to these intervals. When a lower future price is identified, vehicles do not charge for a long time after connection, but are still successfully charged within their target completion time [Fig. 7(b)]. However, in some cases, this means they are not charged by 8 am (although this could be fixed with a tighter charging target). Despite the high peak at 4 am (when the price is low and many vehicles charge) house voltages remain within required limits [Fig. 7(c)] and phase unbalance stays lower than in the uncontrolled case [Fig. 7(d)].

As the cost per kWh figures in Table II show, for the case study presented here greedy charging with price consideration leads to $10 \%$ savings in the cost of charging this set of vehicles, as compared with uncontrolled charging. In this particular 24-h period, the price fluctuations were only minor, and on many days the savings could be considerably greater still (the spot price in Australia can go as high as $\$ 13100 / \mathrm{MWh}$ ).

\section{E. Performance of Linear Constraints}

As discussed in Section VI, the optimization problem as posed in this paper can quickly grow to thousands of decision variables and constraints. To reduce computation time and allow near-real-time computation, our constraints have been linearized (Section IV). This linearization is achieved by assuming a DC-equivalent model of the network (and only real 


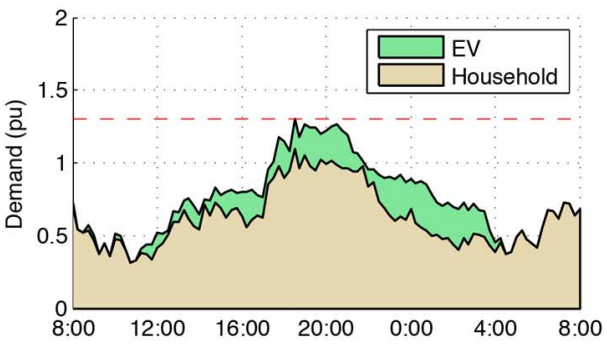

(a)

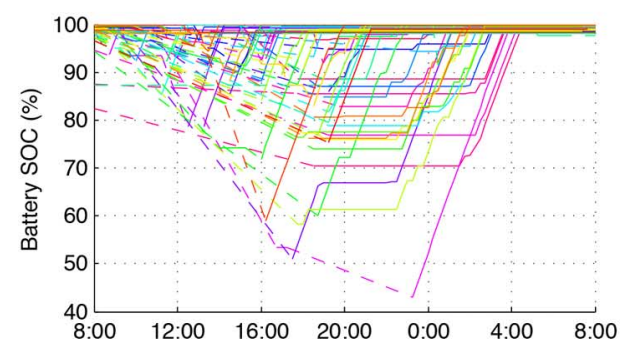

(b)

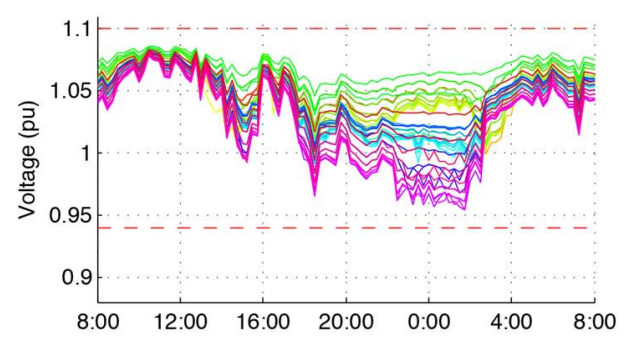

(c)

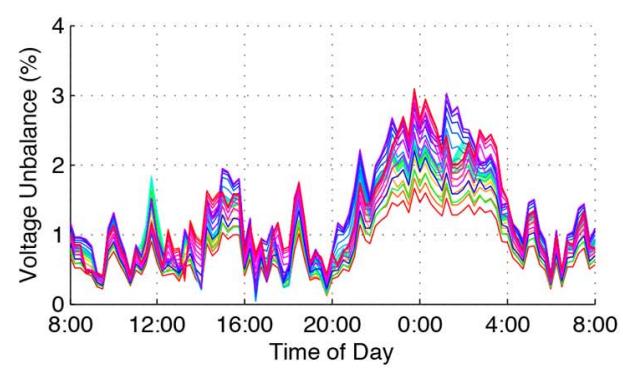

(d)

Fig. 6. Greedy charging. (a) Total demand. (b) Vehicle charging rates. (c) Household voltages. (d) Unbalance.

power demands) when calculating transformer, backbone, service line, and voltage drop constraints. While this significantly reduces our computation time, a drawback of this linearization is that we are only approximating the solution space, and that the computed solution may not in fact be the true, globally optimal one. In this section we explore the cost of this linearization, and discuss how close to the true global optimal solution our approximate optimal solution is.

Since we do not have a globally optimal solution available (we intend to pursue this in future work), we conduct this evaluation by examining at every stage the presently active constraints. Fig. 9(a) shows which constraints are active during the greedy optimization run previously presented in Fig. 6 and discussed in Section V-A. This scenario uses a transformer capacity

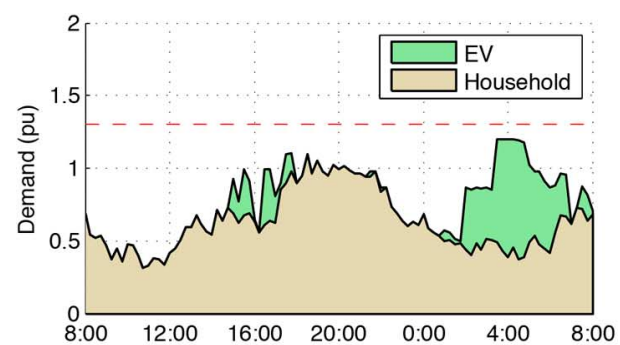

(a)

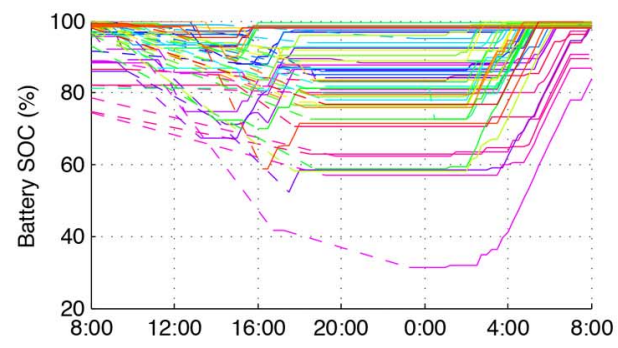

(b)

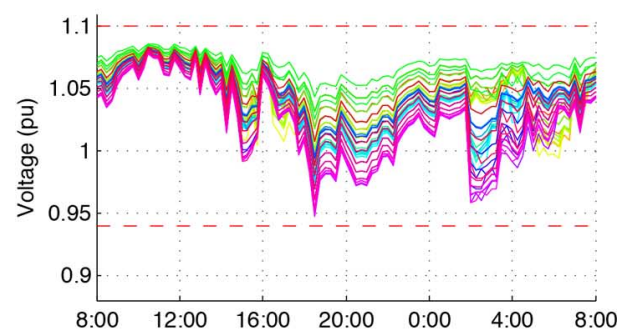

(c)

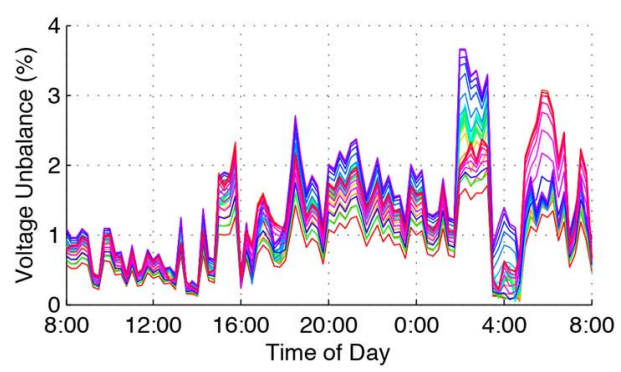

(d)

Fig. 7. Greedy charging with price. (a) Total demand. (b) Vehicle charging rates. (c) Household voltages. (d) Unbalance.

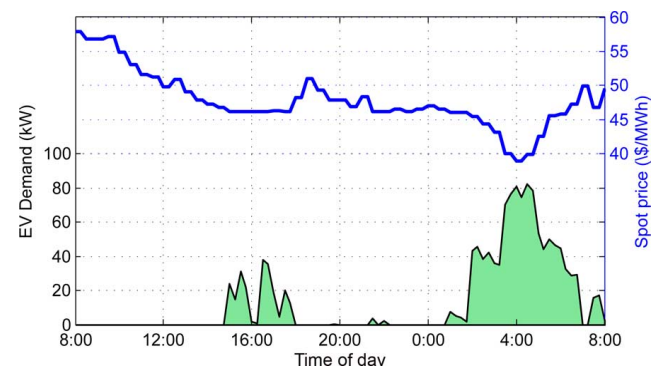

Fig. 8. Effect of spot price on charging demand when using the price-based greedy optimization method.

(denoted "TX" throughout Fig. 9) of $100 \mathrm{KVA}$, a backbone current rating (denoted "BB") of $350 \mathrm{~A}$, a service line rating (denoted "SL") of $100 \mathrm{~A}$, and a minimum allowed voltage (denoted 


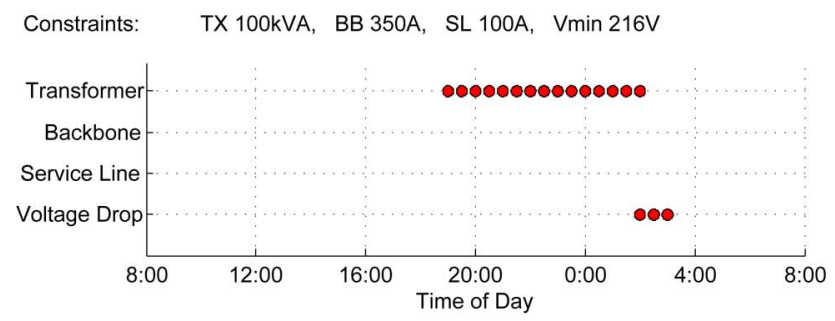

(a)

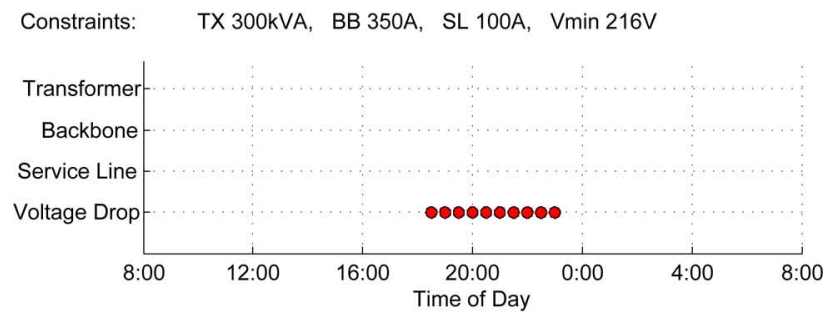

(b)

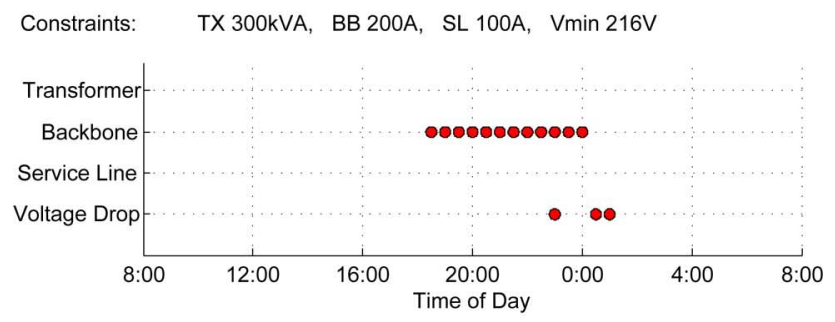

(c)

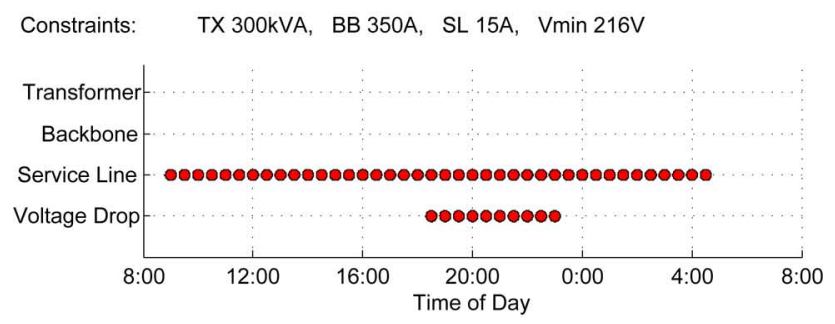

(d)

Fig. 9. Active constraints for four separate scenarios. (a) Active constraints for a scenario in which transformer capacity is the main limitation. (b) Active constraints for a scenario in which voltage drop is the main limitation. (c) Active constraints for a scenario in which the backbone's current rating is the main limitation. (d) Active constraints for a scenario in which the service line's current rating is the main limitation.

"Vmin") of $216 \mathrm{~V}$. As can be seen, the transformer in this case is the limiting factor during peak hours. When transformer capacity is increased, the main limiting factor becomes voltage drop [Fig. 9(b)]. When backbone current rating is decreased, the backbone becomes the main limiting factor [Fig. 9(c)]. Finally, when the service line current rating is decreased, individual service lines become the main limiting factors [Fig. 9(d)].

By examining these four different scenarios, we were able to generate data for each of the four constraints that we are interested in. To examine how these constraints are affected by our linearization, we generated the solution to the optimization

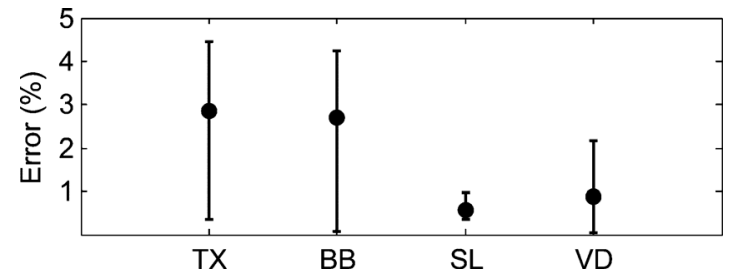

Fig. 10. Constraint errors due to linearization, for transformer capacity (TX), backbone current rating (BB), service line rating (SL), and voltage drop (VD).

problem, then applied the solution to our model and ran a fully complex load flow, and finally compared the outputs of that load flow to the hard limits existing in the network. The error due to linearization was then computed as

$$
\frac{\text { Complex load flow output }- \text { True limit }}{\text { True limit }} \times 100 \% \text {. }
$$

For example, if the active constraint in our linearized optimal solution leads to a minimum voltage at house $H$ of $220 \mathrm{~V}$, but the real limit is $216 \mathrm{~V}$, then the error due to linearization is $1.85 \%$.

Fig. 10 shows the average errors obtained for each of our four constraints, averaged over all four scenarios discussed above. The error bars indicate maximum and minimum error obtained for each constraint at any point during any of the runs.

The greatest errors are obtained for the transformer capacity, with a maximum error of $4.46 \%$. This is due to the fact that the loads in our system are actually inductive, and additional power is required due to reactive power demands that our optimal solution does not consider. However, we constrain demand on each individual phase, so even a slight excess demand on one phase does not lead to an excess of total transformer demand (which we do not see at all in our simulations).

Encouragingly, only small errors were obtained for voltage drop, suggesting that the linearization as described in Section IV-C is indeed an effective way to constrain voltage.

While this does not provide a definitive answer to how close our approximate solution is to the real solution, we consider the errors due to linearization, for the most part less than $3 \%$, to be an effective compromise in return for significant gains in the speed of computation. In addition, the simulation results presented in Section VII-A confirm that the proposed method allows effective charging of vehicles without network constraint violation. We intend to compare these results to fully complex optimal solutions in future work.

\section{F. Maximum Possible Vehicle Uptake}

Both the vehicle profiles that are chosen, and the locations in the network that vehicles are assigned to, can have a big impact on whether a simulation run succeeds or not (for example, loads at the far ends of the network have considerably greater impact on voltage drop than loads near the transformer, see also [19]). In a separate set of simulations, we examined a large number of different configurations having a variety of $\mathrm{EV}$ penetration levels, for each charging algorithm.

Uncontrolled charging could only be sustained in this network to penetration levels of $10-15 \%$. Beyond this, typically low voltage issues caused our runs to fail. For optimal greedy charging (with or without price consideration), all simulation runs up to penetration levels of $80 \%$ succeeded. Beyond this, 
there were instances where no solution to the optimization could be found. We consider this an encouraging result: using centralized, optimal charging, high levels of EV uptake are sustainable without any major infrastructure upgrades being required.

\section{CONCLUSION}

In this paper we expressed electric vehicle charging as a linear optimization problem that takes into account both the present and the anticipated constraints in the distribution network over a finite charging horizon. The solution for the full horizon can be recalculated in discrete intervals, taking into account the dynamic nature of changing demand and vehicle arrival/departure. We implemented two objectives: the first maximizes total charging of all vehicles, and the second minimizes the cost of charging. Our solutions were tested in a validated model of a real network, using demand and vehicle travel data obtained in this network.

The main conclusions of this paper are the following:

- By implementing load control as proposed here, existing networks can sustain high electric vehicle uptake rates, without the need for further infrastructure upgrades. In other words, existing assets may be used much more efficiently, leading to savings for both network operator and consumer. In our case study based on a real network in Melbourne, Australia, uncontrolled vehicle charging can lead to network failures at uptake rates of only $10-15 \%$. With optimal load control, vehicle uptake rates beyond $80 \%$ would be sustainable.

- In the network we studied (where power factor is high), a linear approximation of key network constraints such as transformer capacity, line current ratings, and voltage drop is an effective way to simplify the problem and allow faster and more repeated computation of the solution. Particularly if scaling up to larger networks, or if trying to solve load control problems at e.g., the substation level, such a trade-off in solution accuracy may be worth the gains in computability.

- Price-based optimal charging can effectively schedule vehicle charging during times when price is low, thereby offering significant savings in the cost of charging $-10 \%$ in the case study presented here, and likely more on days which see greater fluctuations in the wholesale electricity price.

In ongoing work, we are considering how to generalize the present analysis and approach to low voltage networks where a significant amount of harmonic distortion exists, and/or where current and voltage waveforms show significant phase shift. We further intend to examine how the unpredictability of spot price and base load demand affect our solution, extend this work to a fully complex, nonlinear $\mathrm{AC}$ formulation, examine the impact of distributed generation, and explore some of the issues surrounding fairness.

\section{ACKNOWLEDGMENT}

The authors would like to thank R. Jegatheesan of United Energy for providing valuable data and advice.

\section{REFERENCES}

[1] U.S. Department of Energy, EV Everywhere: Grand Challenge Blueprint, accessed Aug. 2013 [Online]. Available: http://www1. eere.energy.gov/vehiclesandfuels/electric_vehicles/pdfs/eveverywhere_blueprint.pdf

[2] C. Gerkensmeyer, M. C. W. Kintner-Meyer, and J. DeSteese, Technical Challenges of Plug-In Hybrid Electric Vehicles and Impacts to the US Power System: Distribution System Analysis, Tech. Rep., Jan. 2010

[3] J. Lopes, F. Soares, and P. Almeida, "Integration of electric vehicles in the electric power system," Proc. IEEE, vol. 99, no. 1, pp. 168-183, Jan. 2011.

[4] M. Masoum, P. Moses, and S. Hajforoosh, "Distribution transformer stress in smart grid with coordinated charging of plug-in electric vehicles," in Proc. IEEE Power and Energy Society Conf. Innovative Smart Grid Technologies (ISGT), Jan. 2012

[5] J. de Hoog, D. A. Thomas, V. Muenzel, D. C. Jayasuriya, T. Alpcan, M. Brazil, and I. Mareels, "Electric vehicle charging and grid constraints: Comparing distributed and centralized approaches," in Proc. IEEE Power and Energy Society General Meeting, Jul. 2013.

[6] Z. Ma, D. Callaway, and I. Hiskens, "Decentralized charging control for large populations of plug-in electric vehicles," in Proc. IEEE Int. Conf. Control Applications, Sep. 2010.

[7] L. Gan, U. Topcu, and S. Low, "Optimal decentralized protocol for electric vehicle charging," in Proc. IEEE Conf. Decision and Control and Eur. Control Conf., Dec. 2011.

[8] K. Clement-Nyns, E. Haesen, and J. Driesen, “The impact of charging plug-in hybrid electric vehicles on a residential distribution grid," IEEE Trans. Power Syst., vol. 25, no. 1, pp. 371-380, Feb. 2010.

[9] E. Sortomme, M. M. Hindi, S. D. J. MacPherson, and S. S. Venkata, "Coordinated charging of plug-in hybrid electric vehicles to minimize distribution system losses," IEEE Trans. Smart Grid, vol. 2, no. 1, pp. 198-205, Mar. 2011.

[10] S. Sojoudi and S. H. Low, "Optimal charging of plug-in hybrid electric vehicles in smart grids," in Proc. IEEE Power \& Energy Society General Meeting, 2011.

[11] P. Richardson, D. Flynn, and A. Keane, "Optimal charging of electric vehicles in low-voltage distribution systems," IEEE Trans. Power Syst., vol. 27, no. 1, pp. 268-279, Feb. 2012.

[12] K. Zhan, Z. Hu, Y. Song, Z. Luo, Z. Xu, and L. Jia, "Coordinated electric vehicle charging strategy for optimal operation of distribution network," in Proc. IEEE PES Innovative Smart Grid Technologies Europe, 2012.

[13] Z. Luo, Z. Hu, Y. Song, Z. Xu, and H. Lu, "Optimal coordination of plug-in electric vehicles in power grids with cost-benefit analysis - Part I: Enabling techniques," IEEE Trans. Power Syst., vol. 28, no. 4, pp. 3546-3555, Nov. 2013.

[14] A. D. Giorgio, F. Liberati, and S. Canale, "Electric vehicles charging control in a smart grid: A model predictive control approach," Control Eng. Practice vol. 22, no. 0, pp. 147-162, 2014 [Online]. Available: http://www.sciencedirect.com/science/article/pii/S0967066113001871

[15] Z. Angelovski and K. Handberg, Demand Management of Electric Vehicle Charging Using Victorias Smart Grid, DiUS Computing, Tech. Rep., May 2013.

[16] W. H. Kersting, Distribution System Modeling and Analysis, 3rd ed. Boca Raton, FL, USA: CRC, 2012.

[17] Electricity Distribution Code. Victoria, Australia, accessed May 2012 [Online]. Available: http://www.esc.vic.gov.au/Energy/Distribution/Electricity-Distributioncode

[18] SAE Surface Vehicle Recommended Practice J1772, SAE Electric Vehicle Conductive Charge Coupler, Society of Automotive Engineers Std..

[19] J. de Hoog, V. Muenzel, D. C. Jayasuriya, T. Alpcan, M. Brazil, D. A. Thomas, I. Mareels, G. Dahlenburg, and R. Jegatheesan, "The importance of spatial distribution when analysing the impact of electric vehicles on voltage stability in distribution networks," Energy Syst., pp. 1-22, Apr. 2014 [Online]. Available: http://link.springer.com/article/10.1007\%2Fs 12667-014-0122-8

[20] Victorian Integrated Survey of Travel and Activity, accessed Sep. 2012 [Online]. Available: http://www.transport.vic.gov.au/research/statistics/victorian-integratedsurvey- of-travel-and-activity 


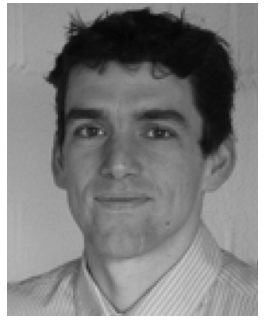

Julian de Hoog (M'12) received the B.Sc. degree in computer science and mathematics from McGill University, Montreal, QC, Canada, and the M.Sc. and $\mathrm{Ph} . \mathrm{D}$. degrees in computer science from the University of Oxford, U.K.

His main research interests are in distribution networks, electric vehicles, smart charging and demand response. He has been a Research Fellow at The University of Melbourne since February 2012.

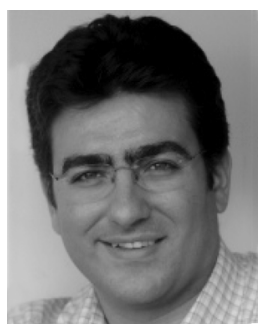

Tansu Alpcan (SM'13) received the M.S. and Ph.D. degrees in electrical and computer engineering from University of Illinois at Urbana-Champaign (UIUC).

He has been a Senior Lecturer at The University of Melbourne, Australia, since October 2011. His main research interests are distributed decision making, game theory, and control with applications to energy markets, smart grid, and demand response. He is the author and editor of the books Network Security: A Decision and Game Theoretic Approach and Mechanisms and Games for Dynamic Spectrum Allocation, respectively, both of which are published by the Cambridge University Press, Cambridge, U.K.

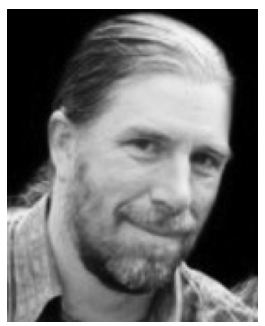

Marcus Brazil received the Ph.D. degree in mathematics from La Trobe University, Melbourne, Australia.

$\mathrm{He}$ is an Associate Professor and Reader at The University of Melbourne, Australia. His main research interests are computational geometry and combinatorial optimization, particularly with applications to network optimization in areas such as VLSI, underground mining, and electric power distribution.

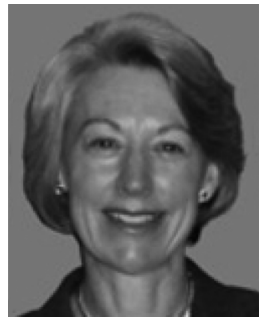

Doreen Anne Thomas (SM'06) received the M.Sc. and D.Phil. degrees (mathematics) from the University of Oxford, U.K.

Her research interests are in network optimization with applications in distribution networks, telecommunications, VLSI design and access to networks in underground mines.

Dr. Thomas is a Fellow of the Australian Academy of Technological Sciences.

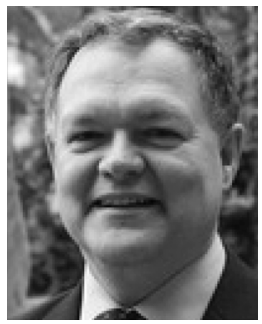

Iven Mareels (F'01) received the ir degree from Gent University, Belgium, in 1982 and the Ph.D. degree in systems engineering from the Australian National University in 1987.

He is presently Dean of the School of Engineering, The University of Melbourne, Australia. His research interests are in large scale systems theory, both engineered systems such as water or electricity distribution networks, and natural systems such as the brain. 


\section{University Library}

\section{- M M I N E R VA A gateway to Melbourne's research publications}

Minerva Access is the Institutional Repository of The University of Melbourne

Author/s:

De Hoog, J;Alpcan, T;Brazil, M;Thomas, DA;Mareels, IMY

Title:

Optimal Charging of Electric Vehicles Taking Distribution Network Constraints Into Account

Date:

2015

Citation:

De Hoog, J., Alpcan, T., Brazil, M., Thomas, D. A. \& Mareels, I. M. Y. (2015). Optimal

Charging of Electric Vehicles Taking Distribution Network Constraints Into Account.

IEEE Transactions on Power Systems, 30 (1), pp.365-375. https://doi.org/10.1109/

TPWRS.2014.2318293.

Persistent Link:

http://hdl.handle.net/11343/55191 